\title{
FLUID FLOWS IN MICROCHANNELS WITH CAVITIES
}

\author{
Zeta Tak For Yu ${ }^{1}$, Yi-Kuen Lee ${ }^{1}$, Man Wong ${ }^{2}$ and Yitshak Zohar ${ }^{3}$ \\ ${ }^{1}$ Department of Mechanical Engineering, ${ }^{2}$ Department of Electrical and Electronic Engineering \\ Hong Kong University of Science and Technology, Clear Water Bay, Kowloon, Hong Kong \\ ${ }^{3}$ Department of Aerospace and Mechanical Engineering, University of Arizona \\ Tucson, AZ 85721, USA
}

\begin{abstract}
Pressure-driven gas and liquid flows through microchannels with cavities have been studied using both experimental measurements and numerical computations. Several microchannels with cavities varying in shape, number and dimensions have been fabricated. One set of microdevices, integrated with sensors on a silicon wafer, is used for flow rate and pressure distribution measurements in gas flows. Another set of microdevices, fabricated using glass-tosilicon wafer bonding, is utilized for visualization of liquid flow patterns.

The cavity effect on the flow in the microchannel is found to be very small, with the mass flow rate increasing slightly with increasing number of cavities. The flow pattern in the cavity depends on two control parameters; it is fully attached only if both the reduced Reynolds number and the cavity number are small. A flow regime map has been constructed, where the critical values for the transition from attached to separated flow are determined. The numerical computations reveal another control parameter, the cavity aspect ratio. The flow in the cavity is similar only if all three control parameters are the same. Finally, the vorticity distribution and related circulation in the cavity are analyzed.
\end{abstract}

Journal of Microelectromechanical Systems, Vol. 14, No. 6, pp.1-14, 2005

DOI: 10.1109/JMEMS.2005.859086 


\section{INTRODUCTION}

The demand for fine control of micro-scale fluid flows, especially in biotechnology applications, has become a central research theme often referred to as microfluidic systems. The advances in micromachining techniques made it possible not only to realize flows in micro configurations but also to carry out micro flow diagnosis [1]. Simple microchannel flow has been studied experimentally and analytically [2], revealing the relative importance of mechanisms such as slip, compressibility and acceleration. However, this is a simple fully-attached, laminar flow with no flow separation. Experiments in more complex microchannels have also been conducted; flows as in uniform but not straight, e.g. bends or branches $[3,4]$, as well as in straight but non-uniform microchannels, e.g. with expansion/contraction sections or constriction elements $[5,6]$. Indeed, the results indicate that flow separation is a possible cause for a pressure loss along a microchannel, but it has not been confirmed experimentally. Hence, in this work, the flow in a microchannel with cavities is examined, experimentally and numerically, not only to determine the effect of the cavities on the microchannel main stream but, in particular, to study the ensuing flow field within the micro cavity.

Much research work has been devoted to study of flow separation in macro scale because of its importance in many practical applications. Similar understanding of flow separation in micro scale is critical for the construction of sophisticated, compact and massive flow networks. The flow in a microchannel with cavities is essentially a flow between two parallel plates separated by a narrow gap similar to Hele-Shaw flow. Following the solution presented by Stokes, two control parameters have been identified for this class of problems: (i) cavity number; the square of the ratio between a vertical and a planar length scale, and (ii) reduced Reynolds number, the product of the cavity number and the classical Reynolds number. Inserting smooth obstacles between the plates such as a cylinder does not result in flow separation. The streamline pattern of the Hele-Shaw flow has been considered as analogous to that of 2-D, potential (irrotational) flow [7]. Experiments indicate that if the reduced Reynolds number exceeds unity, the inertia term cannot be neglected such that the Stokes solution is no longer valid [8].

Cavity inherently is a flow separation triggering element due to its sharp corners and, consequently, it is considered as an energy loss component in internal flow systems [9]. The energy loss depends on the cavity shape and dimensions. O'Brien [10] studied the streamline pattern in several cavities varying in shape, and concluded that the central vortex often oscillates 
in and out of the cavity. Taneda [11] visualized various separated flow patterns in rectangular cavities depending on the cavity aspect ratio. Cochrane et al. [12] presented several flow patterns in cavities, using both experiments and simulations, for Newtonian and non-Newtonian fluid flows. However, all the patterns included flow separation. Shen and Floryan [13] also conducted experiments and simulations of flows in cavities with various configurations demonstrating the development of corner vortices independent of the cavity aspect ratio. Two very different flow patterns were reported to develop in cavities: an attached flow field was observed in the irrotational-flow regime, whereas flow separation occurred in the Stokes flow regime [14]. Kim et al. [15] studied instabilities related to a viscoelastic creeping flow in a cavity. The critical conditions for the onset of flow transitions and the secondary flows generated by the instability were investigated. They reported that increasing the flow rate resulted in a more pronounced asymmetry of the re-circulating region. The flow was observed to be time-dependent at the higher rates with the dividing streamline oscillating in and out of the cavity.

Additional phenomena and applications are associated with multi-cavity channels. Chow [16] sketched three basic types of rough-surface flow. The sketches in fact depicted the flow over multiple cavities with different spacing, with the flow pattern changing as a function of the distance between successive cavities. Taylor [17] experimentally demonstrated that the force required to sustain a fluid motion decreases when the boundary contains grooves within a given range of dimensions. Dullien and Azzam [18] studied the flow rate and pressure gradient in periodically non-uniform capillary tubes. The development of corner vortices in cracks and cavities prompted researchers to introduce the concept of "fluid" walls [19]. Davis [20] considered a more fundamental problem of a "fluid" wall in an unbounded shear flow, which he solved by utilizing a slip velocity model. Romero-Mendez et al. [21] investigated the influence of the distance between fins on the convection of heat using experiments and simulations. HeleShaw flow was observed for small gap distances, while increasing the distance resulted in flow separation and vortex formation.

Most of the research work related to cavities has been on a macro-scale, and very little information is available for flows in micro cavities. Karniadakis and Beskok [22] have shown that in a 2-D, shear-driven grooved microchannel flow, the flow separated and re-circulated in the grooves even for very small Reynolds number. They reported a reduction in the separation zone for slip flows compared to the no-slip flow. The effect of a single or multi cavities on the flow in a microchannel depending on the cavity geometry has not been reported. Furthermore, 
the flow fields in micro cavities, particularly the flow patterns, have not been investigated systematically, and the control parameters have not been quantitatively characterized. These issues will be addressed in this work using both experimental measurements and numerical computations.

\section{EXPERIMENTAL CONSIDERATIONS}

The design and fabrication of the integrated microchannels with the cavities will be described as well as the experimental techniques used in this study.

\section{A. Device design}

A typical cavity structure is composed of a back-to-back expansion and contraction section as in Figure 1. A variety of microchannels with cavities have been designed to study numerous effects. In one set of microchannels, $4000 \times 40 \times 1.5 \mu \mathrm{m}^{3}$ in dimensions, a single cavity was designed to examine the effect of its particular configuration on the flow in the microchannel and in the micro cavity. The single cavity, always at the microchannel center, varied between $L_{c}=40$ to $160 \mu \mathrm{m}$ in length and between $W_{c}=40$ to $800 \mu \mathrm{m}$ in width. Another set of microchannels, $3825 \times 30 \times 1.5 \mu \mathrm{m}^{3}$ in dimensions, was designed to study the number of cavities effect on the flow field. The cavity configuration was kept constant in this set, $L_{c}=W_{c}=15 \mu \mathrm{m}$, while the number of cavities increased from 1 to a maximum of 127; with the cavities placed symmetrically with respect to the channel center at equal intervals. Both sets included integrated pressure sensors designed in a Wheatstone bridge configuration of 4 piezoresistive elements. The sensors are located at distances of $400,1200,2000,2400$, and $3200 \mu \mathrm{m}$ from the inlet. The third set of microchannels, $400 \times 100 \times 20 \mu \mathrm{m}^{3}$ in dimensions, was designed to study the flow pattern with the cavities. A pair of square cavities, varying between $20 \times 20$ to $70 \times 70 \mu \mathrm{m}^{2}$ in area, was placed at each microchannel center. In all three device sets, the height of the cavities has been designed to be equal to the height of the microchannel.

\section{B. Device fabrication}

The microdevices with integrated pressure sensors were fabricated using standard surface micromachining techniques. The fabrication process has been detailed elsewhere [2], and the 
final device cross section is schematically shown in Figure 2(a). As a result of the fabrication processes involved, the measured channel height of the single-cavity devices was $1.6 \mu \mathrm{m}$, while the height of the multi-cavity devices was measured to be $1.7 \mu \mathrm{m}$.

Bulk micromachining techniques were used to fabricate the microdevices designed for flow visualizations due to the designed larger channel height, and a schematics of the final cross section is shown in Figure 2(b). The microchannels and cavities were formed on the front side of a silicon wafer, while inlet/outlet holes were formed on the wafer backside, using deep reactive ion etching (STS DRIE). After stripping the silicon wafer from all intermediate thin films, it was anodically bonded to a cover glass wafer (Pyrex 7740, Corning Inc., NY, USA) allowing optical observation of the flow pattern in the channel and cavities. Pictures of several fabricated devices are shown in Figure 3.

\section{Experimental set-up}

A schematic diagram of the experimental setup is shown in Figure 4. Argon gas was used under driving pressure of up to $350 \mathrm{kPa}$. The mass flow rate was measured by a glass syringe, connected to the device outlet, with a capacity of $10 \mu \mathrm{L}$. The volume flow rate can be calculated by visually measuring the movement of a water meniscus along the marked scale of the syringe as a function of time. With available gas properties, the mass flow rate is deduced. For pressure measurement, each sensor was calibrated prior to experimentation against an external pressure transducer, and the sensitivity was found to be about $0.02 \mathrm{mV} / \mathrm{kPa} / \mathrm{V}$. The flow rate was adjusted by adjusting the pressure at the inlet, while the channel outlet was fixed at the ambient atmospheric pressure. The signal output of the pressure sensors were recorded using a PC; thus, verifying that steady-state flow has been established prior to collecting flow rate and pressure distribution data.

For flow visualization experiments, the devices were mounted on an Olympus IX70 fluorescence microscope. Water mixed with a surfactant sodium dodecyl sulfate (SDS) at 10\% by weight and $0.49 \mu \mathrm{m}$ fluorescent polymer micro-particles source solution at $10 \%$ by weight was used as the working solution. The fluorescence emitting particles were used as tracers for apparent streamline visualization. The size of the particles has been selected in order to maintain a ratio of at least 1:40 compared to the minimum feature size of the tested devices. Such low ratio ensures that the particle responds to a local point force rather than to a global integration of 
momentum exerted by the fluid flow. SDS as a surfactant in the working fluid reduces the chance of particle adhesion to the channel walls. Furthermore, to avoid gas trapping in the cavity, the tested devices were flushed with SDS solution prior to forcing the actual particlesolution through the microchannels. The volumetric flow rate at the inlet was controlled by a syringe pump with flow rate ranging from 0.01 to $125 \mathrm{ml} / \mathrm{hr}$. Video cassette recorder was used to capture the flow patterns in the micro-devices using a QImaging Retiga 1300C thermoelectric cooled CCD camera connected to a microscope. The camera resolution is quite high $(1280 \times 1024)$; however, it is rather slow with 12 frames per second compared to video frame rate CCD (30 fps).

\section{THEORETICAL AND NUMERICAL CONSIDERATIONS}

The control parameter for classifying flows among various regimes is the Knudsen number, $K n$, defined as:

$$
K n=\frac{\lambda}{H}
$$

where $\lambda$ is the gas mean free path, and $H$ is the channel height. The argon mean free path at atmospheric pressure is $0.104 \mu \mathrm{m}$; hence, the outlet Knudsen number for a $1.6 \mu \mathrm{m}$-high microchannel is about $K n_{o}=0.065$. Flow with $0.001<K n<0.1$ is considered to be in the slip regime. Thus, the flow interior is still governed by the continuum equations:

$$
\begin{gathered}
\nabla \cdot(\rho \mathbf{V})=0 \\
\rho \frac{D V}{D t}=-\nabla P+\mu \nabla^{2} \mathbf{V}+\frac{1}{3} \mu \nabla(\nabla \cdot V)
\end{gathered}
$$

but with a velocity slip boundary condition given by:

$$
U_{s}=-\lambda\left(\frac{\partial U}{\partial n}\right)_{w}
$$


where $\rho, \mu$ and $P$ are the fluid density, viscosity and pressure, while $\mathbf{V}$ is the velocity vector with the components $u, v$ and $w$ in $x, y$, and $z$ directions. For gas flow, the equation of state for ideal gas is used, $P=\rho R T ; R$ and $T$ are the specific gas constant and temperature, respectively. The velocity slip, $U_{s}$, is proportional to the velocity gradient normal to the wall $(\partial U / \partial n)$ at the solid/fluid interface. This is the mathematical model used in the numerical computations of gas flows, while no-slip boundary condition is used in liquid flow, utilizing a commercially available finite-volume code CFD-ACE+ (ESI CFD, Inc. Huntsville, AL, USA). The code verification was done for the case of a uniform microchannel, i.e. no cavities, for which a simple analytical solution has been derived [23]. Assuming 2-D, fully-developed flow in a microchannel, the mass flow rate can be estimated as:

$$
Q_{m}=\frac{H^{3} W P_{o}^{2}}{24 R T L \mu}\left[\left(\left(\frac{P_{i}}{P_{o}}\right)^{2}-1\right)+12 K n_{o}\left(\frac{P_{i}}{P_{o}}-1\right)\right]
$$

where $Q_{m}$ is the mass flow rate; $P_{i}$ and $P_{o}$ are the inlet and outlet pressures; $R$ and $T$ are the specific gas constant and flow temperature; while $L$ and $W$ are the channel length and width, respectively. The equation accounts for compressible and slip flow effects but not for nonparabolic velocity and acceleration effects. Pressure losses due to the channel inlet and outlet have also been neglected in the analysis since the channel length is much larger than its height, $L / H>10^{3}$. The corresponding streamwise pressure distribution is given by:

$$
\frac{P(x)}{P_{o}}=-6 K n_{o}+\sqrt{\left(6 K n_{o}+\frac{P_{i}}{P_{o}}\right)^{2}-\left[\left(\frac{P_{i}^{2}}{P_{o}^{2}}-1\right)+12 K n_{o}\left(\frac{P_{i}}{P_{o}}-1\right)\right]\left(\frac{x}{L}\right)}
$$

where $x$ is the distance along the channel from its inlet.

The mass flow rate is used as the criterion for the convergence of the numerical solver. A comparison between the theoretical calculations, $Q_{T}$, and numerical computations, $Q_{N}$, is shown in Figure 5 as a function of the grid size. The grid size was varied in both streamwise, $\delta_{x}$, and cross-stream directions, $\delta_{z}$, and the agreement between the two sets of results was found to be 
within $0.5 \%$ for $L / \delta_{x}>1500$ and $W / \delta_{z}>10$. Thus, the numerical scheme is adequate to handle gas flow in microchannels in the slip regime.

\section{A. Control parameters for the micro cavity flow}

While the flow in the microchannel is fully-attached, the flow in the cavities can be either separated or attached. Similar to Hele-Shaw flow between two parallel plates separated by a narrow gap, the flow is characterized by two conditions: (i) negligible inertia force compared to friction force, $\rho u(\partial u / \partial x)<<\mu\left(\partial^{2} u / \partial y^{2}\right)$, leading to a small reduced Reynolds number $R e^{*}$ :

$$
R e^{*}=\frac{\rho U_{a} L_{c}}{\mu}\left(\frac{H}{L_{c}}\right)^{2}<<1
$$

where $U_{a}$ is a velocity scale for the cavity flow, and (ii) negligible planar viscous terms compared to the cross-stream term, $\left(\partial^{2} u / \partial x^{2}\right)<<\left(\partial^{2} u / \partial y^{2}\right)$, resulting in a small cavity number, $A c$ :

$$
A c=\left(\frac{H}{L_{c}}\right)^{2}<<1
$$

It is important to emphasize that though this ratio appears in the condition for the reduced Reynolds number, it is an independent condition strictly geometric. Although this control parameter has been noted in the literature, its effect has never been explicitly discussed. The cavity flow is driven by the flow in the main microchannel. Therefore, the velocity scale is taken as the average velocity in the microchannel given by: $U_{a}=Q_{m} / \rho A$, where $A$ is the channel crosssectional area. Since $Q_{m}$ and $A$ are readily available not only in the simulations but also in the experiments, both parameters $R e^{*}$ and $A c$ are known for every tested configuration.

\section{CAVITY EFFECT ON THE MICROCHANNEL FLOW}


The effects of single or multi cavities in a microchannel on the flow rate through and pressure distribution along the channel are analyzed using both experimental measurements and numerical computations in comparison with results for a microchannel with no cavity.

\section{A. Mass flow rate}

The argon mass flow rate through a microchannel with a single cavity at the center as a function of the pressure difference between its inlet and outlet is shown in Figure 6(a) for various cavity dimensions. Experimental measurements, full symbols, are presented for a cavity size with a width range of $40-800 \mu \mathrm{m}$ and length range $40-160 \mu \mathrm{m}$ under driving pressure difference up to $350 \mathrm{kPa}$. Numerical computations have been carried out for exactly the same configurations for a driving pressure difference of $240 \mathrm{kPa}$. These results are compared with analytical calculations based on Equation 5, solid curve, for a uniform and straight microchannel $4000 \mu \mathrm{m}$ in length, $40 \mu \mathrm{m}$ in width and $1.6 \mu \mathrm{m}$ in height. The experimental measurements for the channels with a single cavity scatter within $\pm 3 \%$ of the theoretical flow rate calculated for a cannel with no cavity. Error analysis has shown that just a 1\% difference in height will result in a 3\% error in mass flow rate [2]. Not only it is a formidable challenge measuring the height of a fabricated microchannel at a particular cross-section with $\pm 1 \%$ accuracy, but it is even more difficult to ascertain the height uniformity along the channel. Hence, within the experimental error, a single cavity with its tested relative dimensions has a negligible effect on the mass flow rate regardless of its size. Furthermore, the numerical computations for channels with a fixed height of $1.6 \mu \mathrm{m}$ but varying in cavity size are practically the same, confirming that height variations are the likely reason for the scatter in the measurements rather than the variations in cavity dimensions.

A question arises due to this finding about the effect of numerous cavities, rather than a single cavity, on the flow rate through a microchannel. Whether a single cavity has an effect too small to be detected or no effect at all can be addressed by systematically increasing the number of cavities along the channel. For this purpose, several microchannels have been fabricated; each $3825 \mu \mathrm{m}$ in length, $30 \mu \mathrm{m}$ in width and $1.7 \mu \mathrm{m}$ in height. The number of square cavity pairs, distributed uniformly along the channel, varied between $N=1$ and 127 ; each $15 \times 15 \mu \mathrm{m}^{2}$ in area and $1.7 \mu \mathrm{m}$ in height. The maximum number of pairs was selected such that the distance between neighboring cavities is no less than the cavity length. This is in effort to eliminate flow 
interaction between neighboring cavities and, consequently, allowing the assumption that the effect of multi-cavity pairs is additive. The mass flow rate measurements and computations through the tested microchannels are summarized in Figure 6(b). Both sets of results are in good agreement showing slight increase of the mass flow rate, under the same pressure drop, with increasing number of cavity of pairs.

In order to quantify this effect, the mass flow rate through the microchannels with multicavities is normalized using the theoretical flow rate through a microchannel with no cavities. The results are plotted in Figure 7 as a function of the number of cavity pairs for certain pressure drops. The measured and the computed mass flow rate increases almost linearly with the number of cavity pairs, indicating that the accumulative effect of the multi cavities is a linear combination. It seems that the resistance to the flow passing through the cavity region decreases due to the larger cross-sectional area and, consequently, the mass flow rate increases. Moreover, the spacing of the cavities along the channels is large enough resulting in linear effect of the cavity number, analogous to the electrical resistance of a wire consisting of several resistors connected in series. This linear behavior could be the result of the narrow gap between the channel top and bottom surfaces leading to a fully-attached flow. Thus, taking advantage of the numerical scheme, the mass flow rate per unit length through multi-cavity microchannels with exactly the same planar configurations but with infinite channel height, i.e. 2-D flow, has also been computed. Although the flow in the cavity may not be fully-attached, the linear increase of flow rate with increasing number of cavities is similar to the 3-D flow. This suggests that the flow rate increase with the number of cavities is independent of the cavity flow pattern as long as the spacing between neighboring cavities is large. Moreover, even for the microchannel with the maximum number of cavity pairs, $N=127$, the relative increase in the mass flow rate compared to that of a microchannel with no cavity is no less $7 \%$. Utilizing the linear relationship, the relative increase due to a single cavity pair is estimated to be about $0.05 \%$. This explains why the effect of a single cavity on the mass flow rate could not be detected.

\section{B. Streamwise pressure distribution}

Measured and computed streamwise pressure distributions along microchannels with single and multi cavities are compared in Figures 8(a) and (b), respectively, with theoretical calculations for a straight microchannel based on Equation 6. The expected non-linear 
distribution due to the gas compressibility is evident. Consistent with the mass flow rate findings, the effect of either a single cavity or several cavity pairs on the pressure distribution is well within the experimental error; thus, too small to be detected. However, since the pressure gradient is a measure of the local resistance to the flow, the computed distributions could explain the increase in the flow rate with increasing number of cavities.

A blow-up of the computed pressure distribution along the channel symmetry line $(y=z=0)$ at the cavity section is compared in Figure 9 with the calculated distribution for a straight channel with no cavity. Whereas the pressure decreases monotonically in the uniform microchannel, two inflection points are observed in the pressure distribution of the microchannel with cavities. The upstream and downstream points coincide with the start and end of the cavity respectively. Clearly, the pressure gradient decreases and then increases due to the flow divergence into the cavity and convergence back into the microchannel. The main stream enters the cavity and, thus, the effective cross-sectional area increases leading to lower resistance to the flow. The net result is an increase in the mass flow rate of a microchannel with cavities due to local decrease in drag along each cavity. Furthermore, the pressure fluctuations due to the cavities are less than $1 \mathrm{kPa}$; i.e., less than $1 \%$ of the ambient atmospheric pressure. Consequently, only the computations can reveal this local cavity effect, which is smaller than the experimental error estimated to be $\pm 2 \%$.

\section{THE MICRO CAVITY FLOW FIELD}

Applying a pressure difference between the inlet and outlet of a microchannel with cavities results not only in the main stream passing through the microchannels, but also in a complicated flow field within the side cavities. Since it is very difficult to carry out measurements in a micro cavity, the flow field is analyzed mainly by utilizing numerical computations. However, the flow pattern simulations are supported by experimental flow visualizations.

\section{A. Attached and separated flow patterns}

The analysis for Hele-Shaw flow revealed two control parameters: $R e^{*}$ and $A c$, defined in Equations 7 and 8, respectively. Numerical computations of the cavity flow field resulted in attached or separated flow in the cavity depending on the values of these parameters. In order to 
support the simulation results, flow visualization experiments have been conducted in which the value of the control parameters varied systematically. The planar velocity vector plot at the cavity mid-height, $y_{c}=0$, is shown in Figures 10 (a) and (b) for $R e^{*}=2$ and 20, respectively, having the same $A c=0.25$. Flow visualization images are shown for the same parameters in Figure 11 . Evidently, Figures 10(a) and 11(a), the flow is fully attached for the smaller reduced Reynolds number, $R e^{*}=2$. Increasing the reduced Reynolds number to $R e^{*}=20$, Figures $10(\mathrm{~b})$ and $11(\mathrm{~b})$, results in a separated flow inside the cavity although the height is very small, $H \sim 10 \mu \mathrm{m}$. To underline the independent effect of the cavity number, similar simulations and visualizations have been repeated for different values of $A c$ with constant $R e^{*}$, and the results are respectively shown in Figures 12 and 13. Indeed, the flow is fully attached for the lower cavity number, $A c=0.25$, Figures 12(a) and 13(a). However, even for the same very low reduced Reynolds number, $R e^{*}=0.04$, flow separation in the cavity occurs if the ratio is increased to $A c=1$ as shown in Figures 12(b) and 13(b).

The cavity flow pattern is found to undergo transition from attached to separated with increasing either $R e^{*}$ or $A c$, and it is interesting to find the critical values for this transition. In the absence of an adequate analytical model, flow patterns for various combinations of $R e^{*}$ or $A c$ need to be examined. Experimentally, only a limited number of microdevices can be fabricated and tested. However, since the flow pattern simulations were found to be in good agreement with the flow visualizations, the numerical scheme can easily be used for systematically varying both $R e^{*}$ and $A c$ to compute the cavity flow field. All the combinations of $R e^{*}$ and $A c$ used in this investigation are marked in Figure 14. Based on the computed velocity vector plot, as in Figures 10 and 12, each cavity flow pattern is determined to be either attached or separated and, correspondingly, denoted by either hollow or full symbols. This phase-plot diagram can now be considered as a flow regime map with clear boundaries between different flow patterns. The flow in the cavity is fully attached only if two criteria are simultaneously met: $R e^{*<4}$ and $A c<0.327$. Flow separation occurs in the cavity if either $R e^{*}>10$ or $A c>0.327$. Whereas the simple analysis required that both $R e^{*}$ and $A c$ be very small, the computations provide sharp critical values. In order to provide experimental support to these critical values, video clips of flow visualizations have been recorded in a limited number of fabricated devices. A typical example of separated liquid flow is shown in Figure 15. A fluorescent particle is clearly seen rotating in the extracted images, two of which are shown in Figures 15(a) and (b). A periodical path can be re-constructed as in Figure 15(c), indicating a separated flow pattern. The experimental observations, marked in 
Figure 14, are consistent with the computationally found critical values of the control parameters.

B. The cavity aspect ratio

Spanwise profiles of the normalized streamwise velocity, $u\left(z_{c}\right)$, inside the cavity are plotted in Figures 16(a) and (b) for attached and separated flows, respectively. The profiles are along the cavity centerline, $x_{c}=L_{c} / 2$, at the channel mid-plane, $y=y_{c}=0$. The normalizing maximum velocity, $u_{m}$, is always at the channel/cavity interface $z_{c}=0$. The attached flows are calculated for various cavity dimensions varying between 40 to $160 \mu \mathrm{m}$ in both length and width, while the height is fixed at $1.6 \mu \mathrm{m}$. Although the profiles exhibit non-trivial trends, Figures 16(a), they collapse into two distinct groups. The common characteristic of each group is a cavity aspect ratio defined as:

$$
A r \equiv \frac{W_{c}}{L_{c}}
$$

which varies from 0.5 to 2 . This implies that the cavity aspect ratio is another control parameter such that cavity flow fields with the same $A r$ are similar. Cavities with larger aspect ratio are wider; hence, less momentum is transported from the channel/cavity interface, $z_{c} / W_{c}=0$, into the cavity edge, $z_{c} / W_{c}=1$. Consequently, the normalized streamwise velocity continuously decreases with increasing $A r$.

In order to investigate the effect of the location or number of cavities on the flow in the cavity, the computations have been repeated for microchannels with single and multi-cavity pairs under separated flow conditions, Figure 16(b). The separated flow is characterized by flow reversal inside the cavity as the direction of the streamwise velocity changes from positive to negative around $z_{c} / W_{c}=0.27$. The profiles for the first, middle and last cavity of the microchannel with 127 cavity pairs collapse together with the profile for the microchannel with a single cavity pair. This indicates a developed self-similar flow pattern within all cavities with negligible interaction between neighboring cavities, and the flow inside the cavity is dominated by the main stream properties at the cavity section. This is consistent with the linear increase of the mass flow rate with increasing number of cavities. Flow similarity is then obtained for either attached or separated cavity flow as long as the cavity aspect ratio $A r$ is the same, independent of the location and number of cavities in a microchannel. 


\section{Cross-stream vorticity}

Cavity flow separation, accompanied by flow reversal, occurs if either $R e^{*}>10$ or $A c>0.327$. A vortex-like motion develops within the re-circulation zone inside the cavity as seen in Figures 10(b) and 12(b). This secondary flow is counter-rotating with respect to the main flow and, hence, can be characterized by using vorticity. The cross-stream vorticity at mid-plane $y_{c}=0$, related to the planar velocity distribution, is given by:

$$
\Omega_{y}\left(x_{c}, z_{c} ; y_{c}=0\right)=\left.\left(\frac{\partial u}{\partial z}-\frac{\partial w}{\partial x}\right)\right|_{y_{c}=0}
$$

Typical cross-stream vorticity distributions at cavity mid-plane, $y=0$, for attached and separated flow, due to increasing either the reduced Reynolds number or the cavity number, are plotted in Figure 17. Two sharp positive peaks are the dominant feature of the attached flow distribution in Figure $17(\mathrm{a}), A c=0.08$ and $R e^{*}=0.05$, due to the sharp counter-clockwise turns the flow undergoes near the corners upon entering and exiting the cavity. The flow in the most part of the cavity area rotates in the opposite direction, clockwise, in a potential-like pattern. Consequently, the magnitude of the vorticity associated with this motion is practically zero since, away from the corners, the motion inside the cavity is almost irrotational. The vorticity distributions for the separated flow, due to increasing either $R e^{*}$ to 50 (Fig. 17b) or $A c$ to 2.9 (Fig. 17c), are very different. The re-circulation zone, with counter-clockwise flow, occupies most of the cavity. The vortex-like motion in the cavity is nearly irrotational with negligible positive vorticity. The two positive vorticity peaks are still present due to the counter-clock rotation near the cavity corner. However, unlike the attached flow, the two peaks are not as prominent since they are bridged by a narrow region of slightly lower vorticity. This vorticity is the result of the shear layer formed at the interface between the main stream in the microchannel and the re-circulation zone inside the cavity. Furthermore, two regions with considerable negative vorticity appear in the separated flow as a result of the flow along the cavity side walls near the two corners. 


\section{Cavity circulation}

The vorticity distribution is found to be complicated with no sharp quantitative difference between attached and separated flow. The planar vorticity distribution shown in Figure 17 can be conveniently represented by the single-value circulation function defined as:

$$
\Gamma_{c}=\Gamma\left(y_{c}=0\right)=\left.\int_{0}^{L_{c}} d x_{c} \int_{-W / 2}^{W_{c}} \Omega_{y}\right|_{y_{c}=0} d z_{c}
$$

$\Gamma_{c}$ is in essence the flux of the cross-stream vorticity through the cavity mid-plane area. The total circulation can be decomposed into its positive, $\Gamma_{c}^{+}$, and negative component, $\Gamma_{c}^{-}$, respectively plotted in Figures 18(a) and (b) as a function of $R e^{*}$ for various values of $A c$. Interestingly, each set of curves, for either the positive or negative circulation, collapse together into a single curve for $A c<0.18$. Moreover, both collapsed curves exhibit different behavior depending on whether $R e^{*}$ is smaller or larger than 10 , corresponding to attached or separated flow. In particular, the negative circulation is nearly zero as long as the flow is attached, $R e^{*}<10$, and it increases in magnitude with the reduced Reynolds number when the flow separation takes place, $R e^{*}>10$.

The total circulation can be rendered non-dimensional using the velocity and length scales, $U_{a}$ and $L_{c}$, as follows:

$$
\Gamma^{*}=\frac{L_{c}}{U_{a} A_{c}} \Gamma_{c}
$$

where $A_{c}=L_{c}\left(W_{c}+W / 2\right)$ is the integration area in Equation 11. The dependence of the normalized circulation $\Gamma^{*}$ on $R e^{*}$ is shown in Figure 19 with $A c$ as the varying parameter. The nondimensional circulation collapses into a single curve for cavities with a very small gap, $A c<0.05$. For cavities with $A c>0.05$, the normalized circulation increases with respect to the single curve with increasing $A c$. Depending on $R e^{*}$, similar to Figure 18, two distinct regions are visible along the curve corresponding to attached and separated flow pattern. Both regions can be curvefitted with empirical formula as follows: 


$$
\begin{gathered}
\Gamma^{*}=0.0035 \ln \left(R e^{*}\right)+0.41 \quad \text { for } \quad R e^{*}<6 \\
\Gamma^{*}=0.012 \ln \left(R e^{*}\right)+0.4 \quad \text { for } \quad R e^{*}>6
\end{gathered}
$$

This means that as long as the cavity height is small compared to its planar length scale, i.e. $H / L_{c}<0.2$, the normalized circulation is a function of only the reduced Reynolds number for both the attached, $R e^{*}<6$, and the separated flow regime, $R e^{*}>6$. Following the Hele-Shaw flow approximation [24], resembling a potential flow solution, the circulation around any closed curve lying in a horizontal plane must be zero. Clearly, the cavity total circulation is not zero even for attached flow due to the dominant viscous effect.

\section{CONCLUSIONS}

Gas and liquid flows in microchannels with cavities have been studied utilizing both experimental and numerical techniques. One set of devices was fabricated on silicon wafers with integrated sensors for gas pressure measurements, and another set was fabricated using glass-tosilicon wafer bonding for liquid flow visualization. Rectangular cavities varying in dimensions, location and number have very small effect on the flow in the microchannel. The mass flow rate increases almost linearly with the number of cavities, with an increase of about $7 \%$ for the maximum number of 127 cavity pairs. On the other hand, the minute local decrease of the pressure gradient in the cavity section, observed in the computations, is well within the experimental error and cannot be measured. Hence, whether the cavity flow is attached or separated, the cavity reduces the local resistance to the flow either due to larger cross-sectional flow area or lower friction.

The flow pattern in the cavity is governed by two control parameters: the reduced Reynolds number, $R e^{*}$, and the cavity number, $A c$. The reduced Reynolds number is a product of the classical Reynolds number and the cavity number, while the cavity number is the square of the ratio between the cavity height and length. Based on simulations and supported by visualizations, two independent conditions are required for the cavity flow to be fully-attached: $R e^{*}<4$ and $A c<0.327$. If either $R e^{*}>10$ or $A c>0.327$ flow separation takes place in the cavity regardless of the value of the other parameter. Hence, flow separation can occur even for 
$R e^{*}<<1$, if the cavity height is sufficiently large compared to its length. Separation can also occur even for extremely small gap, $A c<<1$, if the reduced Reynolds number is sufficiently high.

The computations further reveal that the cavity flow can be self-similar, whether attached or separated, if not only both $R e^{*}$ and $A c$ are the same but also the cavity aspect ratio $A r$, the ratio between the cavity width and length, is the same. The analysis of the computed cavity circulation shows two distinct functional relationship with the reduced Reynolds number corresponding to attached and separated flow patterns. The normalized circulation converges into a single curve for very small cavity height, $A c<0.05$; thus, depending only on the reduced Reynolds number. Empirical formulas, derived using curve fitting method, show a log-type functional relationship for both the attached and the separated flow regimes.

\section{ACKNOWLEDGEMENTS}

This work is supported by the Hong Kong Research Grant Council through RGC grant HKUST6017/02E and HKUST I2MS01/02.EG08.

\section{REFERENCES}

[1] C.M. Ho, and Y.C. Tai, "Micro-electro-mechanical systems (MEMS) and fluid flows," Ann. Rev. Fluid Mech., vol. 30, pp. 579-612, 1998.

[2] Y. Zohar, W.Y. Lee, S.Y.K. Lee, L. Jiang, and P. Tong, "Subsonic gas flow in a straight and uniform microchannel," J. Fluid Mech., vol. 472, pp. 125-51, 2002.

[3] S.Y.K. Lee, M. Wong, M., and Y. Zohar, "Gas flow in microchannels with bends," $J$. Micromech. Microeng.,vol. 11, pp. 635-644, 2001.

[4] S.Y.K. Lee, Z.T.F. Yu, M. Wong, and Y. Zohar, "Gas flow in a microdevice with a mixing layer configuration," J. Micromech. Microeng., vol. 12, pp. 96-102, 2002.

[5] W.Y. Lee, M. Wong, and Y. Zohar, "Microchannels in series connected via a contraction/expansion section,” J. Fluid Mech., vol. 459, pp. 187-206, 2002.

[6] W.Y. Lee, M. Wong, and Y. Zohar, "Pressure loss in constriction microchannels," $J$. Microelectromech. Syst., vol. 11, pp. 236-244, 2002.

[7] F.S. Sherman, Viscous flow: McGraw-Hill, 1990.

[8] J.A. Liggett, Fluid mechanics: McGraw-Hill, 1994. 
[9] D.S. Miller, Internal flow systems: BHR Group Limited, 1990.

[10] V. O'Brien, "Closed streamlines associated with channel flow over a cavity," The Physics of Fluids, vol. 15, pp. 2089-97, 1972.

[11] S. Taneda, "Visualization of separating Stokes flows," J. of the Physical Society of Japan, vol. 46, pp. 1935-42, 1979.

[12] T. Cochrane, K. Walters, and M.F. Webster, "On Newtonian and non-Newtonian flow in complex geometries," Phil. Trans. R. Soc. Lond. A, vol. 301, pp. 163-181, 1981.

[13] C. Shen, and J.M. Floryan, "Low Reynolds number flow over cavities," Phys. Fluids, vol. 28, p. $3191,1985$.

[14] C. Pozrikidis, Little book of streamlines: Academic Press, 1999.

[15] J.-H. Kim, A. Oztekin, and S. Neti, "Instabilities in viscoelastic flow past a square cavity," J. Non-Newtonian Fluid Mech., vol. 90, pp. 261-81, 2000.

[16] V.T. Chow, Open-channel hydraulics: McGraw-Hill, 1959.

[17] G.I. Taylor, "A model for the boundary condition of a porous material. Part 1," J. Fluid Mech., vol. 49, pp. 319-26, 1971.

[18] F.A.L. Dullien, and M.I.S. Azzam, "Flow rate-pressure gradient measurements in periodically nonuniform capillary tubes," AIChE J., vol. 19, pp. 222-9, 1973.

[19] R.L. Panton, Incompressible flow: Wiley, 1995.

[20] A.M.J. Davis, "Periodic blocking in parallel shear or channel flow at low Reynolds number," Physics of Fluids A, vol. 5, pp. 800-809, 1993.

[21] R. Romero-Mendez, M., K.T. Yang, and R. McClain, "Effect of fin spacing on convection in a plate fin and tube heat exchanger," Int. J. Heat Mass Transfer, vol. 43, pp. 39-51, 1999.

[22] G. Karniadakis, and A. Beskok, Micro flows: fundamentals and simulation: SpringerVerlag, 2002.

[23] E.B. Arkilic, M.A. Schmidt, and K.S. Breuer, "Gaseous slip flow in long microchannels," $J$. Microelectromech. Syst., vol. 6, pp. 167-178, 1997.

[24] D.J. Acheson, Elementary fluid dynamics: Oxford University Press, 1995. 


\section{LIST OF FIGURES}

Figure 1. A schematic illustration of a microchannel with a single cavity.

Figure 2. Schematic cross-sections of the fabricated microchannels for: (a) gas flows using surface, and (b) liquid flow using bulk micromachining techniques.

Figure 3. Pictures of fabricated microchannels with (a) a single cavity, and (b) multi-cavity pairs (127) for gas flows; and (c) a single cavity pair for liquid flow visualizations.

Figure 4. A schematic illustration of the experimental set-up for gas flow rate and pressure distribution measurements as well as liquid flow visualizations.

Figure 5. The convergence of the numerical scheme as a function of the streamwise and spanwise grid-size, based on the comparison between the computed, $Q_{N}$, and theoretically calculated flow rate, $Q_{T}$.

Figure 6. Measured and computed mass flow rate dependence on the total pressure drop in microchannels with: (a) a single cavity varying in dimensions, and (b) multi-cavity pairs varying in number, compared with theoretically calculated flow rate in a uniform microchannel.

Figure 7. The effect of the number of cavity pairs along a microchannel on the measured and computed (3-D \& 2-D) mass flow rate for different total pressure drop.

Figure 8. Measured and computed streamwise pressure distributions for various total pressure drops in microchannels with: (a) a single cavity varying in dimensions, and (b) multicavity pairs, compared with theoretically calculated distributions in a uniform microchannel.

Figure 9. A zoom-in comparison between the computed streamwise pressure distribution in a multi-cavity microchannel and the theoretical distribution along the symmetry line of a straight uniform microchannel $(y=z=0)$; the cavity location is marked by vertical dash lines.

Figure 10. Numerical computations; gas flow pattern dependence on the reduced Reynolds number for the cavity number $A c=0.25$ : (a) attached flow at $R e^{*}=2$, and (b) separated flow at $R e^{*}=20$. [Configuration: $L=357 \mu \mathrm{m}, W=14 \mu \mathrm{m}, H=7 \mu \mathrm{m}$, and $L_{c}=W_{c}=14 \mu \mathrm{m}$ ]

Figure 11. Flow visualizations; liquid flow pattern dependence on the reduced Reynolds number for the cavity number $A c=0.25$ : (a) attached flow at $R e^{*}=2$, and (b) separated flow at $R e^{*}=20$. [Configuration: $L=400 \mu \mathrm{m}, W=100 \mu \mathrm{m}, H=20 \mu \mathrm{m}$, and $L_{c}=W_{c}=40 \mu \mathrm{m}$ ] 
Figure 12. Numerical computations; gas flow pattern dependence on the cavity number for the reduced Reynolds number $R e^{*}=0.04$ : (a) attached flow at $A c=0.25$, and (b) separated flow at $A c=1$. [Configuration: $L=357 \mu \mathrm{m}, W=14 \mu \mathrm{m}, H=7$ (a) or $14 \mu \mathrm{m}$ (b), and $\left.L_{c}=W_{c}=14 \mu \mathrm{m}\right]$

Figure 13. Flow visualizations; flow pattern dependence on the cavity number for the reduced Reynolds number $R e^{*}=0.04$ : (a) attached flow at $A c=0.25$, and (b) separated flow at $A c=1$. [Configuration: $L=400 \mu \mathrm{m}, W=100 \mu \mathrm{m}, H=20 \mu \mathrm{m}$, and $L_{c}=W_{c}=40$ (a) or $20 \mu \mathrm{m}$ (b)]

Figure 14. Flow regime map indicating the regions of attached and separated cavity flow pattern based on the control parameters $A c$ and $R e^{*}$, as determined from both experiments (triangles) and simulations (circles). [attached flow - hollow symbols; separated flow - filled symbols]

Figure 15. Visualization of separated flow in a micro cavity for $R e^{*}=0.17$ and $A c=1$ : (a) and (b) instantaneous images at different times with a tracked particle at different locations; (c) re-construction of the particle periodic path in the re-circulation zone extracted from a series of images.

Figure 16. Numerical computations of the mid-height, $y=0$, spanwise distributions of the streamwise velocity along the cavity center $\left(x_{c} / L_{c}=0.5\right)$ for: (a) single-cavity microchannel with different $A r$, and (b) multi-cavity microchannel with the same $A r=1$ but different $R e^{*}$ and $A c$.

Figure 17. Computed mid-plane distribution of the cross-stream vorticity, $\Omega_{y}$, for: (a) attached, $A c=0.08 \& R e^{*}=0.05$; (b) separated flow, $A c=0.08 \& R e^{*}=50$; and (c) separated flow, $A c=2.9 \& R e^{*}=0.05$.

Figure 18. Mid-plane cavity circulation dependence on the reduced Reynolds number with the cavity number as the varying parameter decomposed into the: (a) positive, and (b) negative component. [hollow symbols - fully attached; filled symbols - separated flow]

Figure 19. Mid-plane, normalized cavity circulation dependence on the reduced Reynolds number with the cavity number as the varying parameter, fitted with empirical formulas (solid lines). [hollow symbols - fully attached; filled symbols - separated flow] 


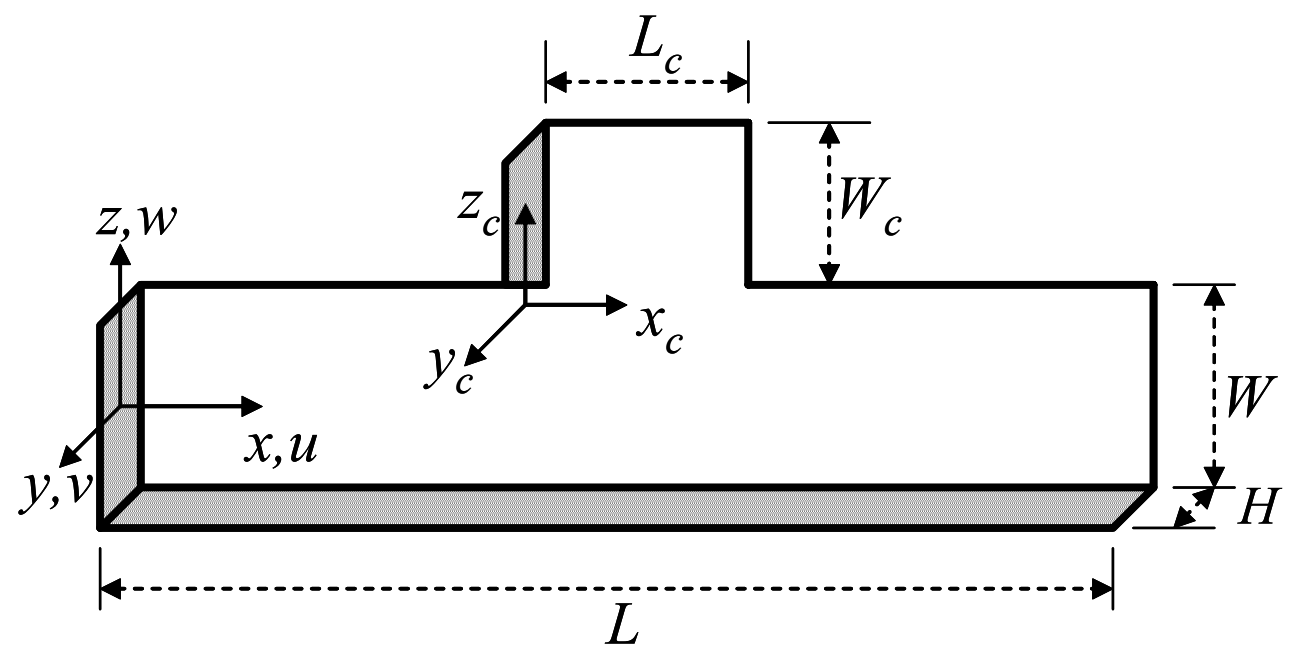

Figure 1: A schematic illustration of a microchannel with a single cavity. 


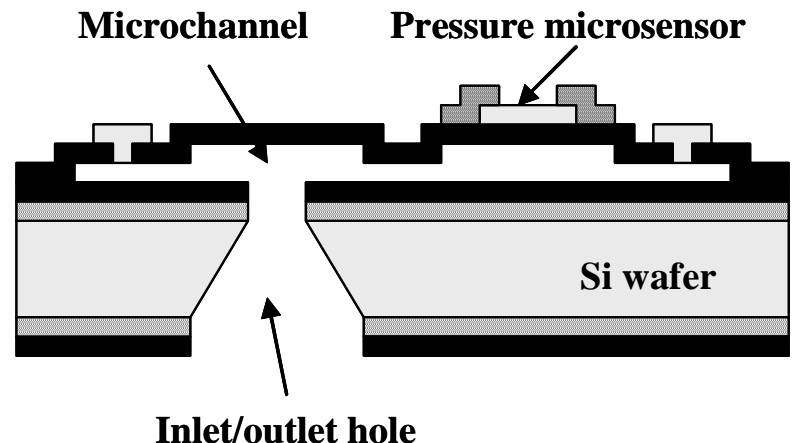

(a)

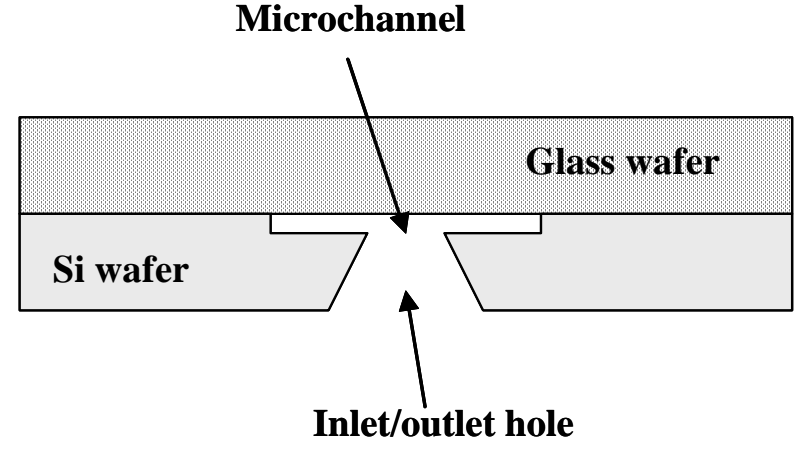

(b)

Figure 2: Schematic cross-sections of the fabricated microchannels for: (a) gas flows using surface, and (b) liquid flow using bulk micromachining techniques. 


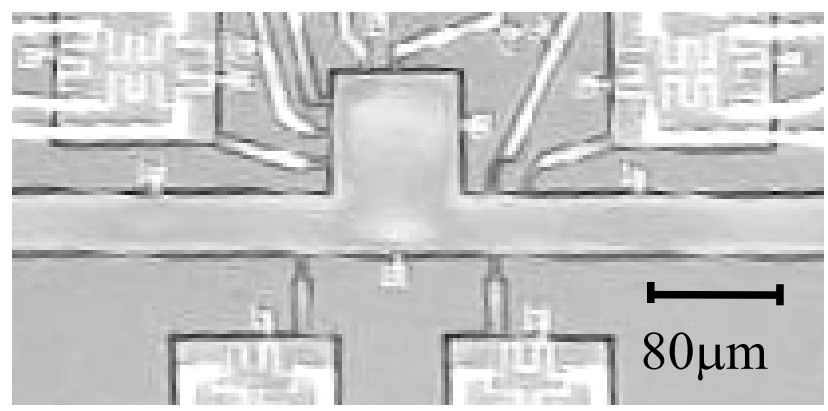

(a)

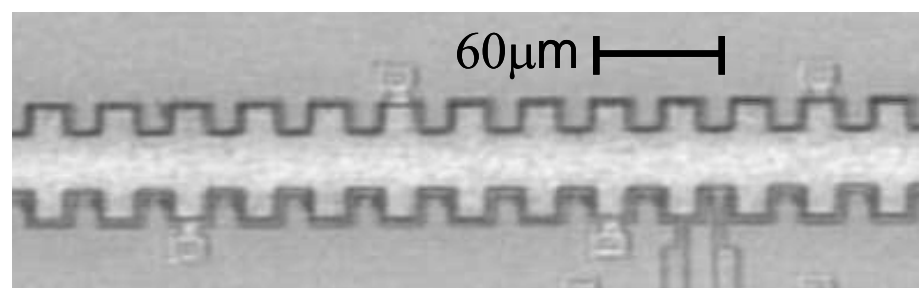

(b)

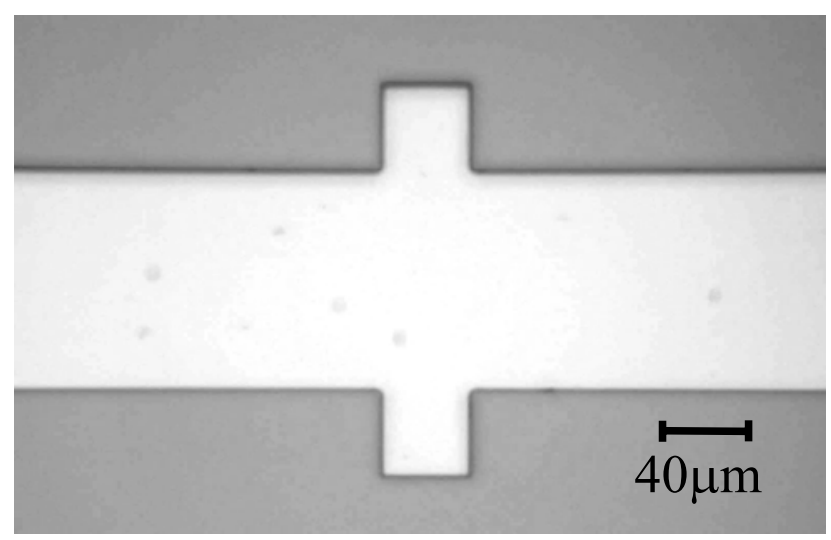

(c)

Figure 3: Pictures of fabricated microchannels with (a) a single cavity, and (b) multi-cavity pairs (127) for gas flows; and (c) a single cavity pair for liquid flow visualizations. 




Figure 4: A schematic illustration of the experimental set-up for gas flow rate and pressure distribution measurements as well as liquid flow visualizations. 


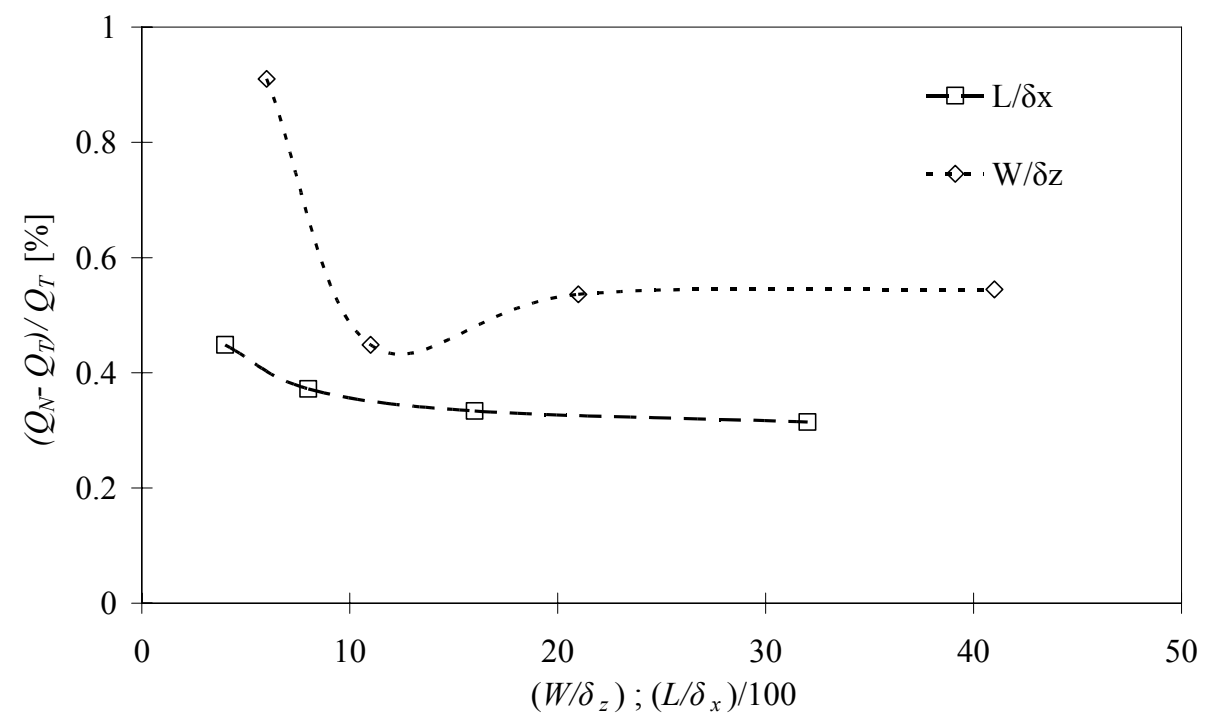

Figure 5: The convergence of the numerical scheme as a function of the streamwise and spanwise grid-size, based on the comparison between the computed, $Q_{N}$, and theoretically calculated flow rate, $Q_{T}$. 


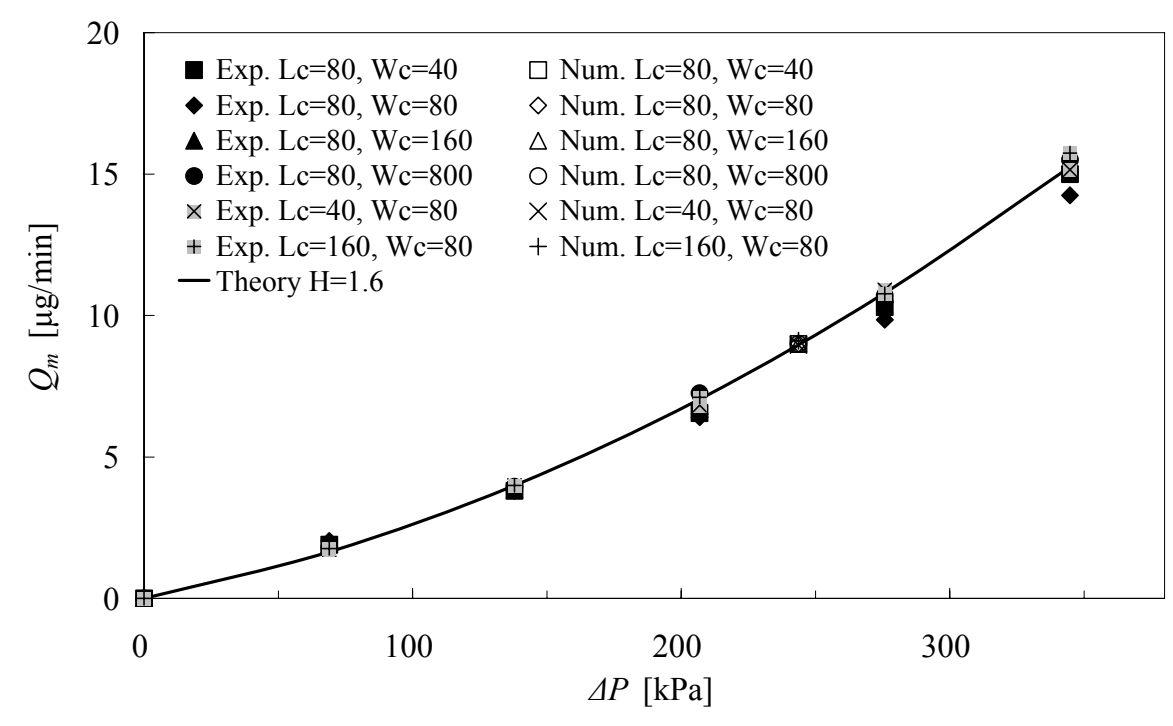

(a)

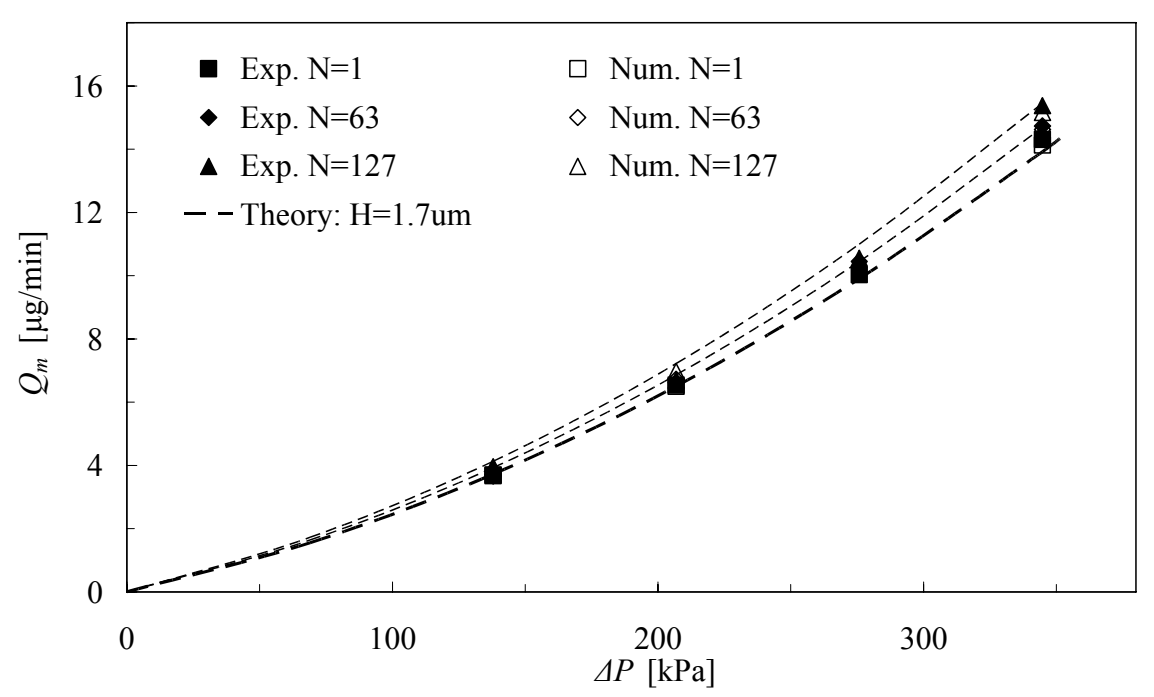

(b)

Figure 6: Measured and computed mass flow rate dependence on the total pressure drop in microchannels with: (a) a single cavity varying in dimensions, and (b) multi-cavity pairs varying in number, compared with theoretically calculated flow rate in a uniform microchannel. 




Figure 7: The effect of the number of cavity pairs along a microchannel on the measured and computed (3-D \& 2-D) mass flow rate for different total pressure drop. 


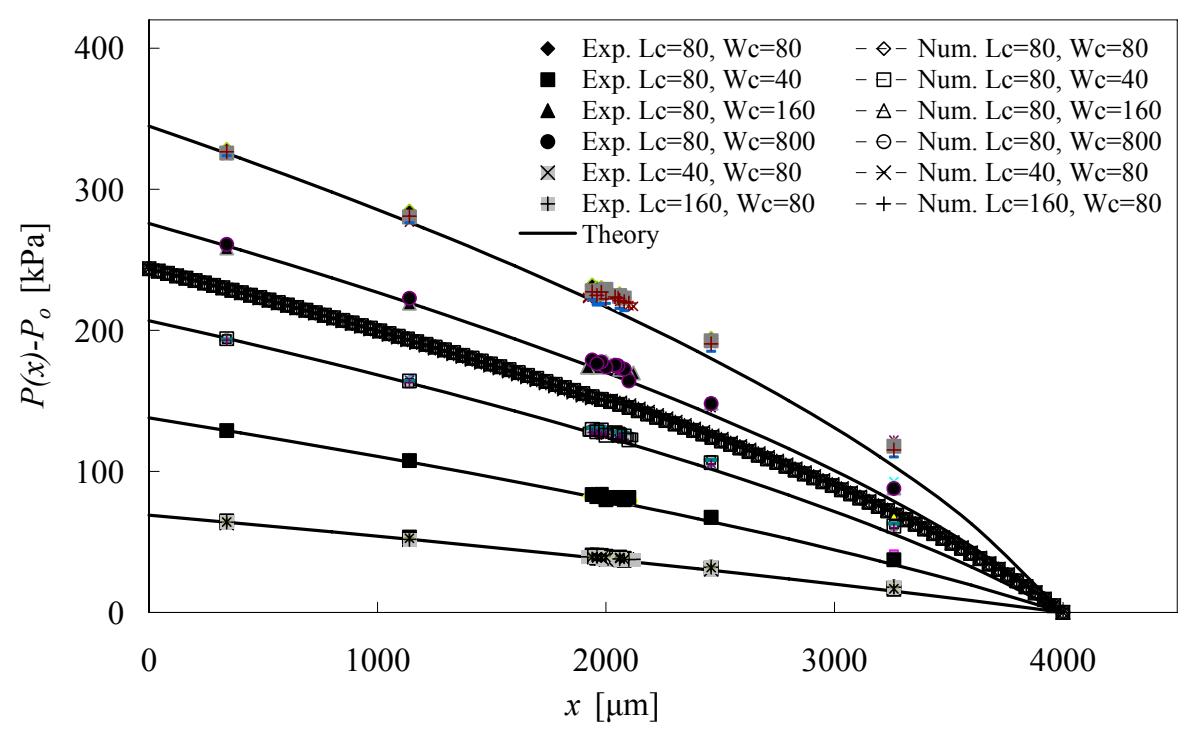

(a)

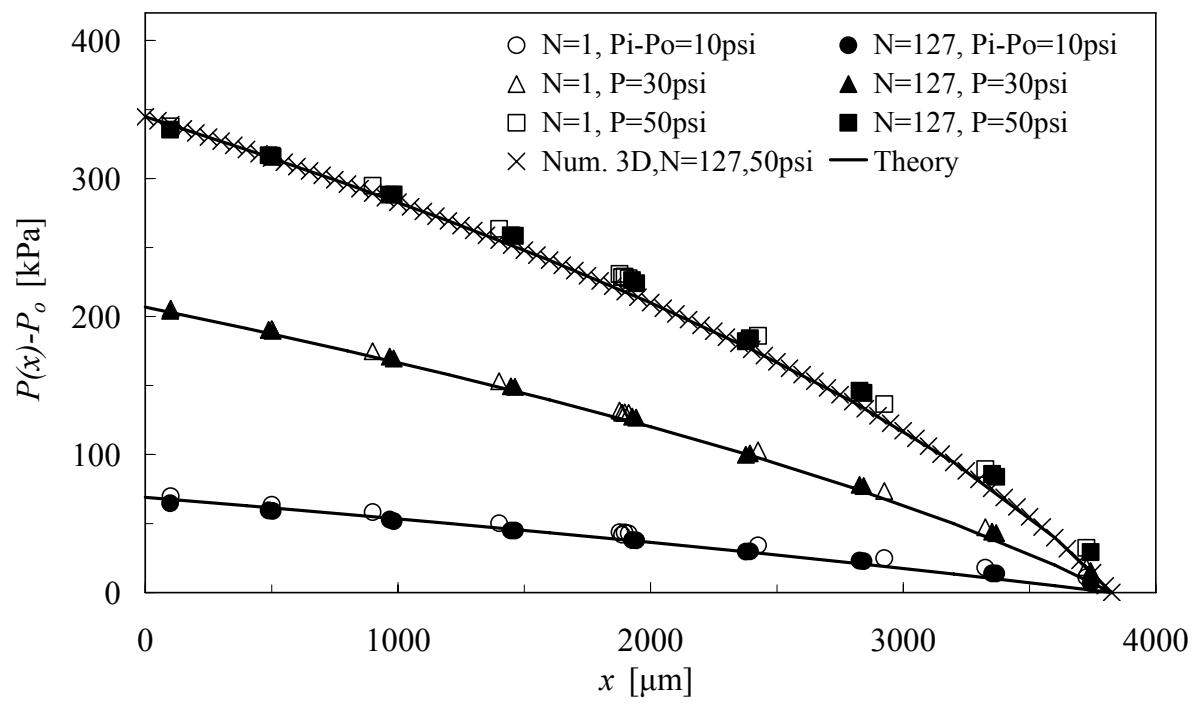

(b)

Figure 8: Measured and computed streamwise pressure distributions for various total pressure drops in microchannels with: (a) a single cavity varying in dimensions, and (b) multi-cavity pairs, compared with theoretically calculated distributions in a uniform microchannel. 


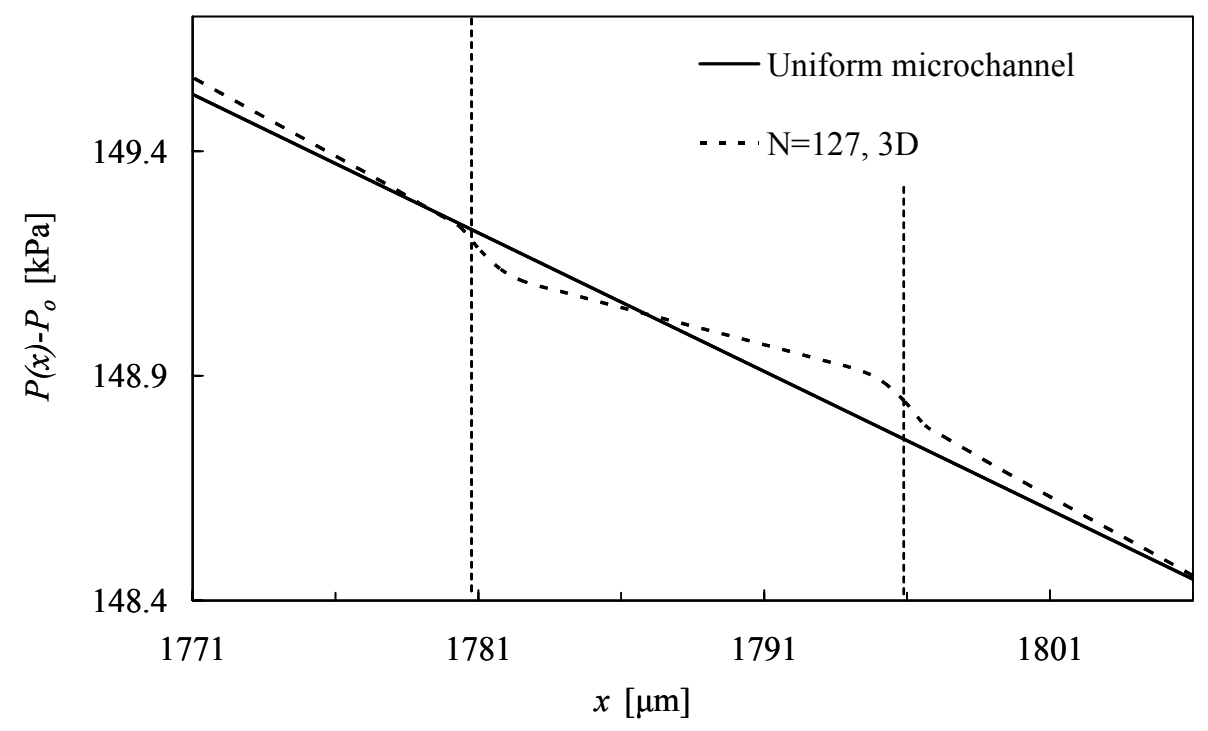

Figure 9: A zoom-in comparison between the computed streamwise pressure distribution in a multi-cavity microchannel and the theoretical distribution along the symmetry line of a straight uniform microchannel $(y=z=0)$; the cavity location is marked by vertical dash lines. 


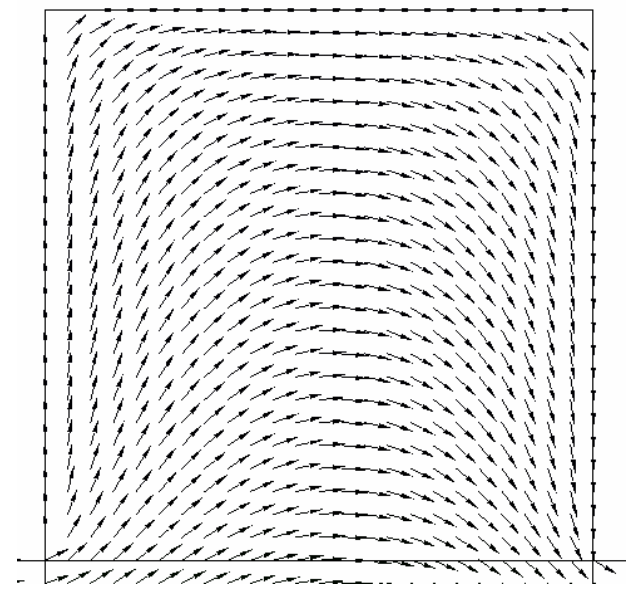

(a)

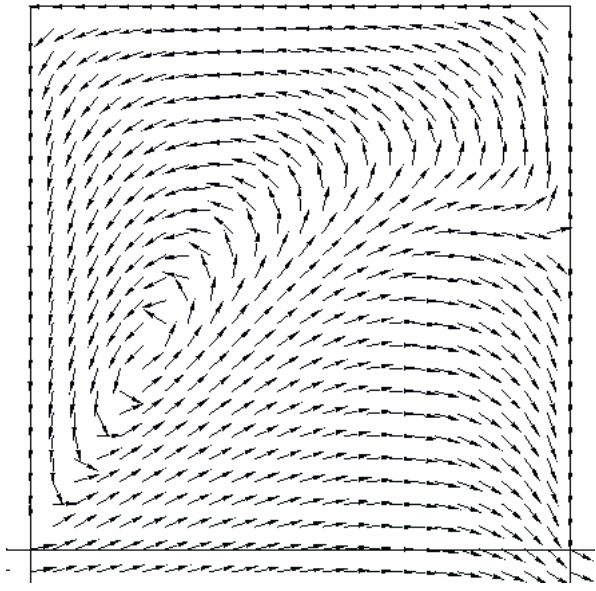

(b)

Figure 10: Numerical computations; gas flow pattern dependence on the reduced Reynolds number for the cavity number $A c=0.25$ : (a) attached flow at $R e^{*}=2$, and (b) separated flow at $R e^{*}=20$. [Configuration: $L=357 \mu \mathrm{m}, W=14 \mu \mathrm{m}, H=7 \mu \mathrm{m}$, and $L_{c}=W_{c}=14 \mu \mathrm{m}$ ]

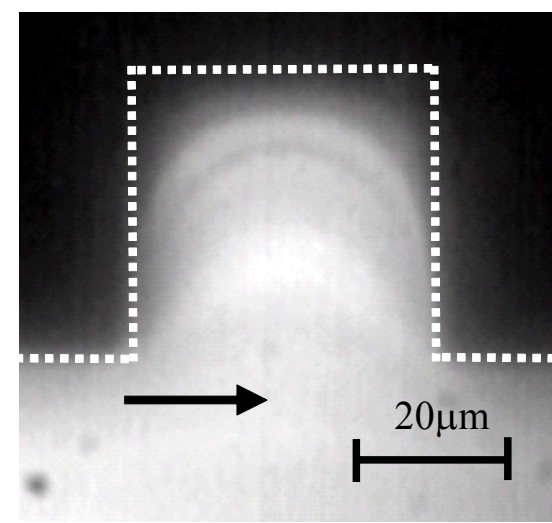

(a)

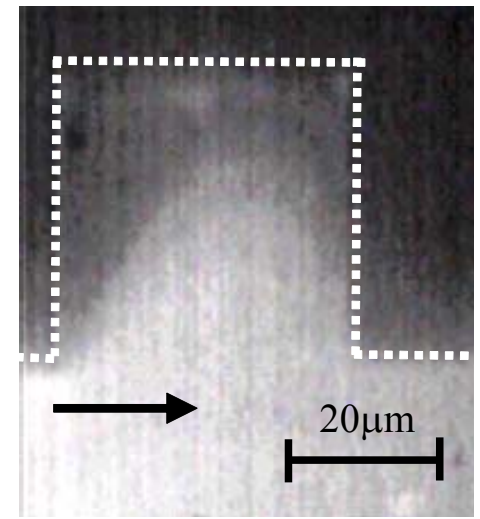

(b)

Figure 11: Flow visualizations; liquid flow pattern dependence on the reduced Reynolds number for the cavity number $A c=0.25$ : (a) attached flow at $R e^{*}=2$, and (b) separated flow at $R e^{*}=20$. [Configuration: $L=400 \mu \mathrm{m}, W=100 \mu \mathrm{m}, H=20 \mu \mathrm{m}$, and $L_{c}=W_{c}=40 \mu \mathrm{m}$ ] 




(a)



(b)

Figure 12: Numerical computations; gas flow pattern dependence on the cavity number for the reduced Reynolds number $R e^{*}=0.04$ : (a) attached flow at $A c=0.25$, and (b) separated flow at $A c=1$. [Configuration: $L=357 \mu \mathrm{m}, W=14 \mu \mathrm{m}, H=7$ (a) or $14 \mu \mathrm{m}$ (b), and $L_{c}=W_{c}=14 \mu \mathrm{m}$ ]

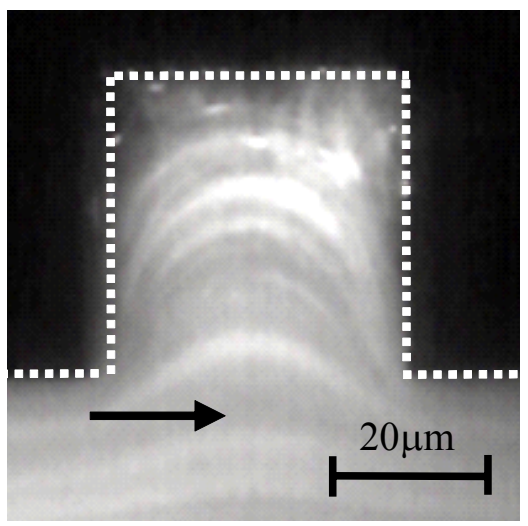

(a)



(b)

Figure 13: Flow visualizations; flow pattern dependence on the cavity number for the reduced Reynolds number $R e^{*}=0.04$ : (a) attached flow at $A c=0.25$, and (b) separated flow at $A c=1$. [Configuration: $L=400 \mu \mathrm{m}, W=100 \mu \mathrm{m}, H=20 \mu \mathrm{m}$, and $L_{c}=W_{c}=40$ (a) or $20 \mu \mathrm{m}$ (b)] 


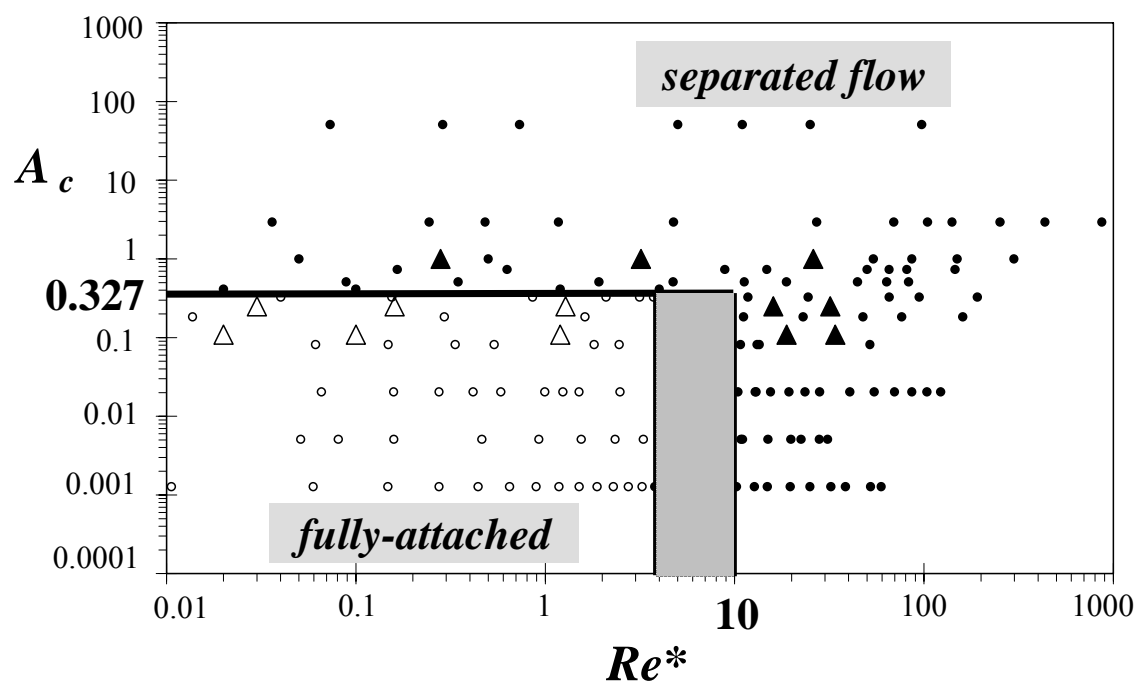

Figure 14: Flow regime map indicating the regions of attached and separated cavity flow pattern based on the control parameters $A c$ and $R e^{*}$, as determined from both experiments (triangles) and simulations (circles). [attached flow - hollow symbols; separated flow - filled symbols] 


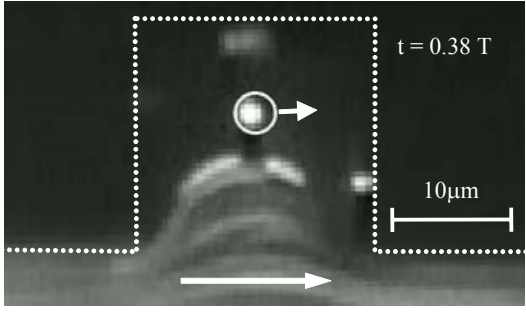

(a)

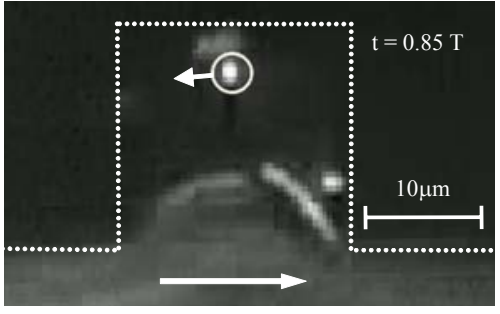

(b)

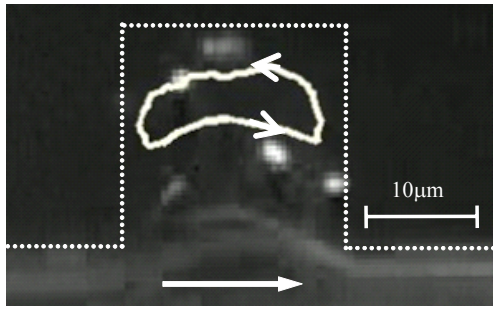

(c)

Figure 15: Visualization of separated flow in a micro cavity for $R e^{*}=0.17$ and $A c=1$ : (a) and (b) instantaneous images at different times with a tracked particle at different locations; (c) reconstruction of the particle periodic path in the re-circulation zone extracted from a series of images. 


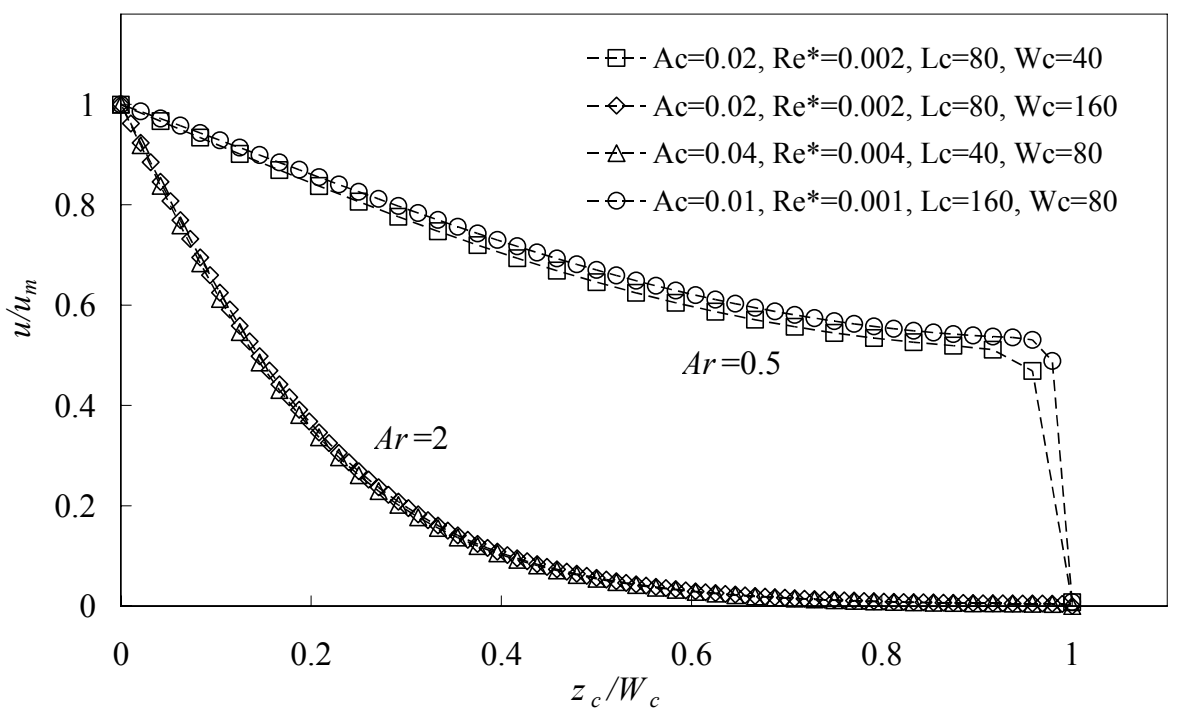

(a)

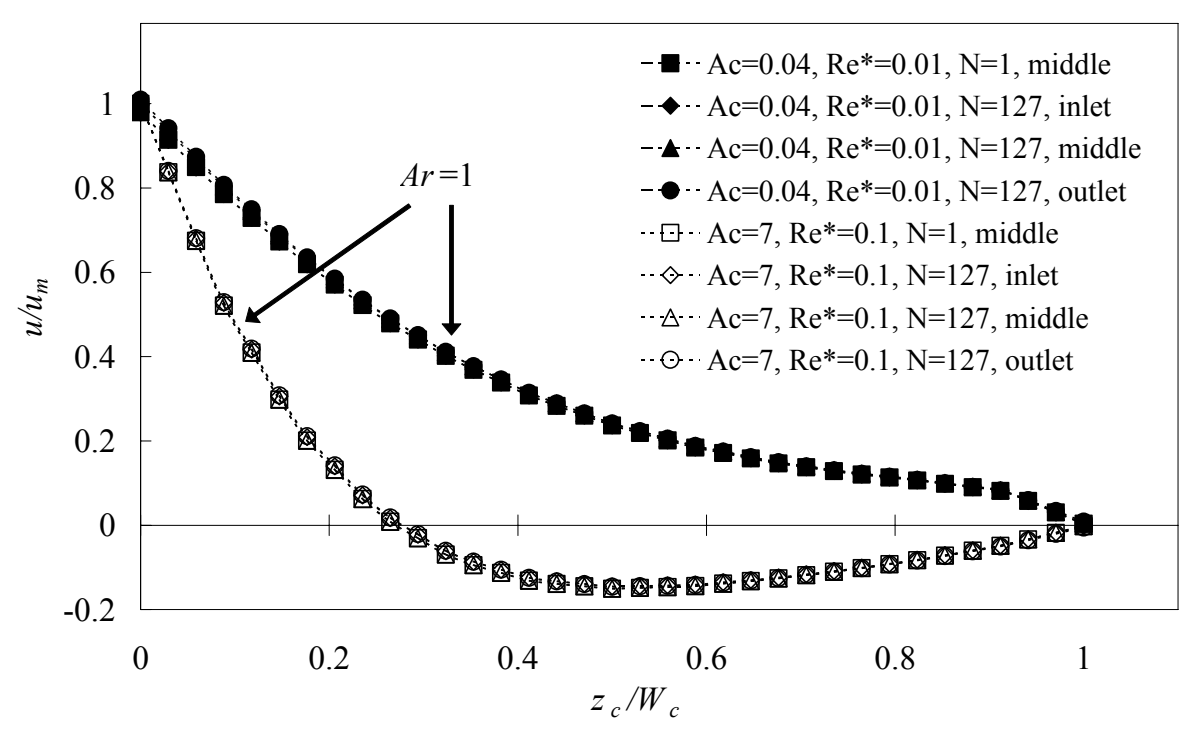

(b)

Figure 16: Numerical computations of the mid-height, $y=0$, spanwise distributions of the streamwise velocity along the cavity center $\left(x_{c} / L_{c}=0.5\right)$ for: (a) single-cavity microchannel with different $A r$, and (b) multi-cavity microchannel with the same $A r=1$ but different $R e^{*}$ and $A c$. 


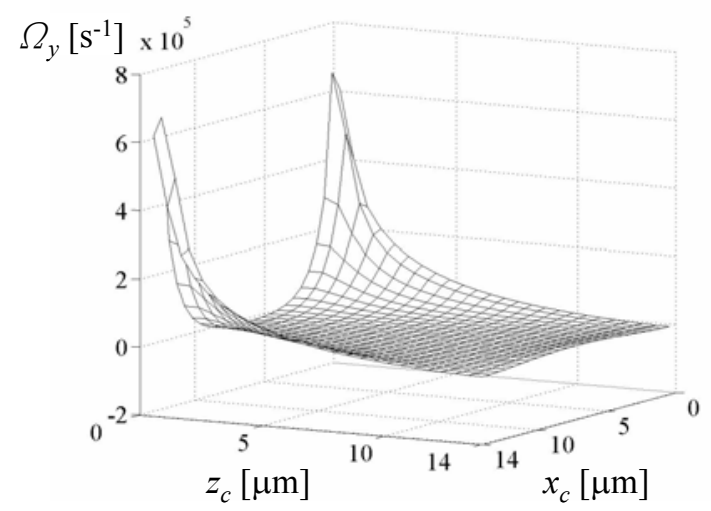

(a)

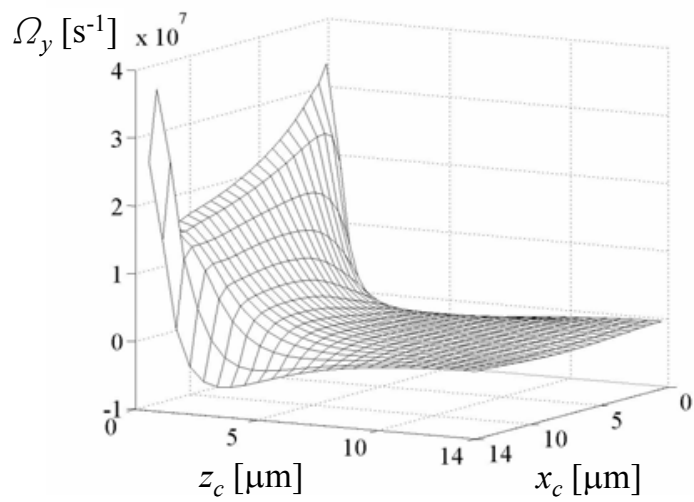

(b)

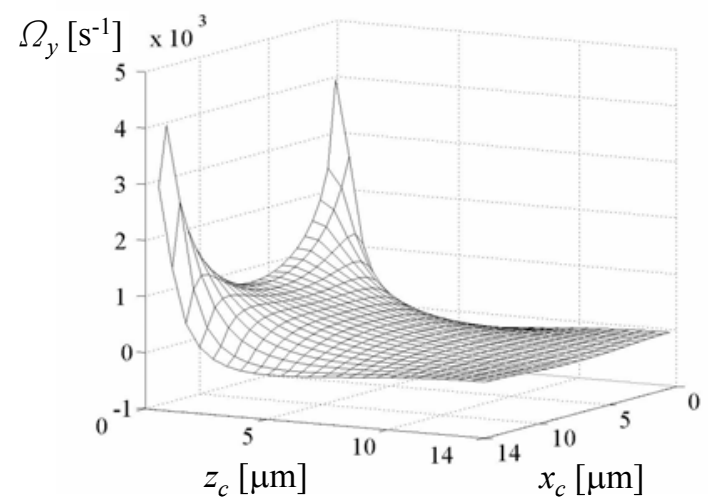

(c)

Figure 17: Computed mid-plane distribution of the cross-stream vorticity, $\Omega_{y}$, for: (a) attached, $A c=0.08 \& R e^{*}=0.05$; (b) separated flow, $A c=0.08 \& R e^{*}=50$; and (c) separated flow, $A c=2.9 \&$ $R e^{*}=0.05$. 


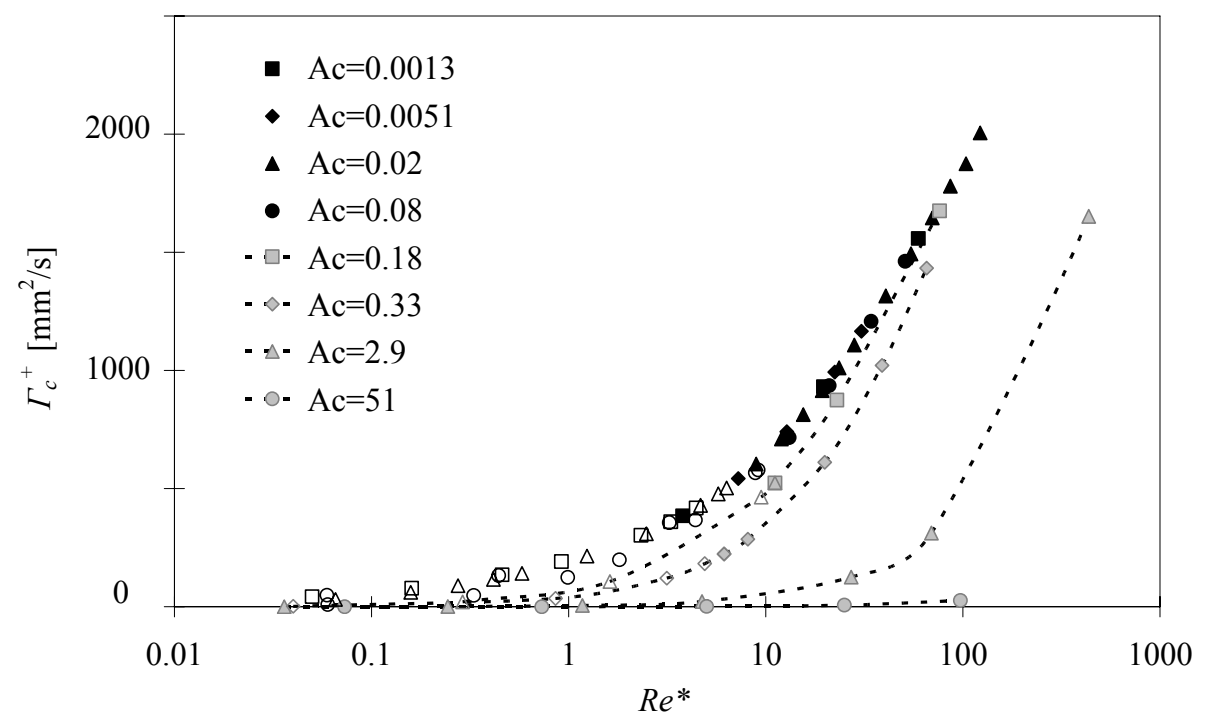

(a)

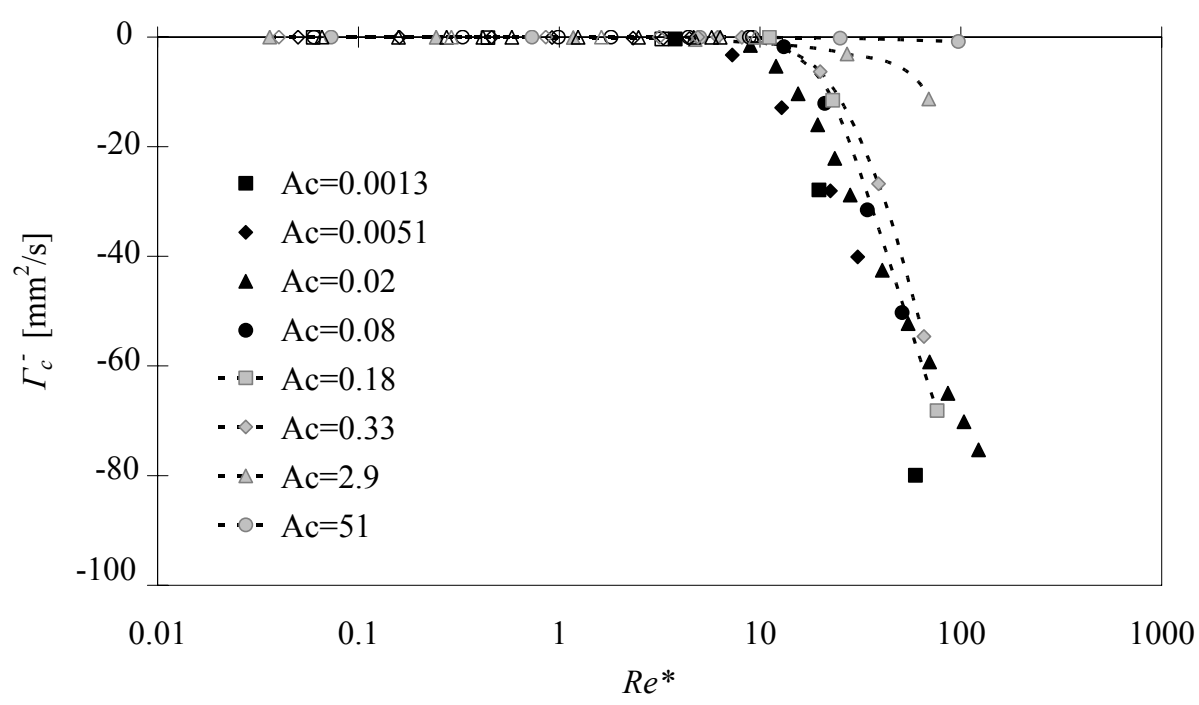

(b)

Figure 18: Mid-plane cavity circulation dependence on the reduced Reynolds number with the cavity number as the varying parameter decomposed into the: (a) positive, and (b) negative component. [hollow symbols - fully attached; filled symbols - separated flow] 




Figure 19: Mid-plane, normalized cavity circulation dependence on the reduced Reynolds number with the cavity number as the varying parameter, fitted with empirical formulas (solid lines). [hollow symbols - fully attached; filled symbols - separated flow] 